\title{
Effect of Tannin Concentration on Mycelial Growth and Development of Auricularia Cornea
}

\section{Zhenyu Zhao}

Jilin Agricultural University

Dongyu Fan

Jilin Agricultural University

\section{Chen Zhang}

Jilin Agricultural University

\section{Fenghua Tian}

Jilin Agricultural University

\section{Bing Song}

Jilin Agricultural University

Chuanwen Jia

Jilin Agricultural University

Changtian Li ( $\sim$ lct@jlau.edu.cn )

Jilin Agricultural University

Yu Li

Jilin Agricultural University

\section{Original article}

Keywords: Auricularia cornea, tannin stress, mycelial morphology, antioxidant enzymes

Posted Date: September 4th, 2020

DOI: https://doi.org/10.21203/rs.3.rs-64350/v1

License: (c) (i) This work is licensed under a Creative Commons Attribution 4.0 International License.

Read Full License 


\section{Effect of tannin concentration on mycelial growth and development}

\section{of Auricularia cornea}

Zhenyu Zhao ${ }^{1}$, Dongyu Fan ${ }^{1}$, Chen Zhang ${ }^{1}$, Fenghua Tian ${ }^{1}$, Bing Song ${ }^{1}$, Chuanwen Jia ${ }^{1}$,

Changtian $\mathrm{Li}^{1,2^{*}}$ and $\mathrm{Yu} \mathrm{Li}^{1,2}$

${ }^{1}$ Engineering Research Center of Edible and Medicinal Fungi, Ministry of Education, Jilin Agricultural University, Changchun 130118, China.

${ }^{2}$ International Cooperative Research Center of China for New Germplasm Breeding of Edible Mushroom, Ministry of Science and Technology.

Address all correspondence to: Changtian Li, Engineering Research Center of Edible and Medicinal Fungi, Ministry of Education, Jilin Agricultural University, Changchun 130118, China; 1ct@jlau.edu.cn 


\section{Abstract}

It is proved that the effect of tannin concentration on the mycelial growth of fungi. Auricularia cornea (white strain) were treated with different concentrations of tannins in Petri-dishes and liquid shaking flasks. The growth rate of mycelium, the ratio of clamp connection, morphology of mycelium, biomass of mycelium and activity of antioxidant enzymes were determined. The results showed that different concentrations of tannin significantly decreased the rate of mycelium. However hign concentration of tannin stress increased the content of thiobarbituric acid in mycelia and had obvious time and concentration effects. Different antioxidant enzyme activities also had different responses to tannin stress. It provides theoretical basis for fungi to utilize and develop substrates containing tannin such as pine.

Key words: Auricularia cornea, tannin stress, mycelial morphology, antioxidant enzymes 


\section{Introduction}

Edible fungi are a valuable source of nutritional and bioactive elements and are used for nutritive and medicinal purposes for thousands of years. Auricularia cornea (white) edible fungi is one of them, having considerable nutritive value and this species has been commonly cultivated and commercialized in China (Cheo, 1982; Wang et al.,2019). Auricularia cornea (white strain), as a new edible fungus species is a cultivated variety with specificity, stability, and consistency obtained by polyspore self-crossing of Auricularia cornea (black strain). This species is highly praised by consumers due to high edible and medicinal value and excellent commodity characteristics. With the rapid development of the Auricularia cornea, the problem of the cultivation material oak is limited.

China's abundant forest resources have always been the strong support of edible fungi cultivation raw materials, especially coniferous forests with Pinaceae as the construction species are rich forest resources in China, and are also the main timber species in China's industrial and agricultural production (Ren 1980, Xu 1990). More than 10 million tons of pine and fir sawdust are produced annually in China, and the tannins contained in this sawdust have a strong inhibitory effect on the growth of edible fungi (He and Wang 1996). Therefore, pine and fir sawdust can not be directly used as cultivation materials for edible fungi, but only as waste burned or discarded naturally which can not pollute the environment. By studying the correlation between tannin in pine sawdust and physiological indexes of Auricularia cornea mycelium, the effet of tannin on Auricularia cornea was preliminarily clarified, which provided a new possibility for cultivation of Auricularia cornea in pine sawdust. 


\section{Materials and methods}

\section{Materials}

Auricularia cornea strain was provided by the Microbial Ecology Laboratory of College of Traditional Chinese Medicine, Jilin Agricultural University.

\section{Strain activation and treatment}

Auricularia cornea strains were inoculated on PDA medium with a $5 \mathrm{~mm}$ diameter perforator for 7 days at $25^{\circ} \mathrm{C}$, and then the hyphae were cultured on petri-dish.

\section{Mycelial culture of Auricularia cornea}

The PDA solid medium contained potato (peeled) $200 \mathrm{~g}$, glucose $20 \mathrm{~g}$, agar $20 \mathrm{~g}$, water $1000 \mathrm{~mL}$. Afterwards, in clean bench, the appropriate amount of tannin mother liquor (sterilized, concentration $5 \mathrm{~g} / \mathrm{L}$ ) was added to the flask to make the tannin (T0200; Sigma Aldrich) concentration in the medium 0, 0.05, 0.1, 0.15, 0.2, 0.25 and $0.3 \mathrm{~g} / \mathrm{L}$, respectively. Then $20 \mathrm{~mL}$ of liquld media was poured into $9 \mathrm{~cm}$ petri-dishes and repeated 5 times each treatment. After solidification of the medium, draw a ' + ' line on the bottom of the petri-dish, the same age mycelium were collected along the edge of activated Auricularia cornea colony with a $5 \mathrm{~mm}$ diameter perforator, and then inoculated at the intersection of ' + ' line. The mycelium was cultured in dark at $25^{\circ} \mathrm{C}$ for 8 days.

\section{Measurement of mycelial growth rate}

The first line was drawn after the second day of germination of Auricularia cornea hyphae, and the second line was drawn on the tenth day. The distance between the two lines was measured, divided by the interval days, and the average daily growth rate of the hyphae was calculated.

\section{Observation on the micromorphology of mycelia}

The liquid strain of $200 \mu \mathrm{L}$ Auricularia cornea was dripped into the plate. The 
method of dilution coating was adopted. After uniform coating, 5-8 aseptic cover slides were inserted at 45 degrees. Each treatment was repeated three times. The hyphae were cultured in the incubator at $25{ }^{\circ} \mathrm{C}$ until the hyphae crawled $2 / 3$ of the cover slides. The cover glass was taken out and observed under the fluorescence microscope and photographed.

\section{Sample fluorescence staining}

For the preparation of the fixed solution: $120 \mathrm{~mL}$ formaldehyde concentrated solution, $4 \mathrm{~g}$ sodium dihydrogen phosphate, $13 \mathrm{~g}$ hydrogen phosphate was added in $1000 \mathrm{~mL}$ distilled water.

For the drying treatment,we took out the cover slide with tweezers, wipe off the negative mycelium and fixed it in the fixing solution for 1 hour, then take it out and dry. The cover slides were washed with PBS buffer for 3-5 times and stained with 2 drops of DAPI dye for 10 minutes. Then rinse with PBS buffer for 1 minute.

\section{Observation}

After treatment, the samples were observed and counted under fluorescence microscope, and the number of clamp connection, mycelia, the diameter, length, surface area and mycelium branching rate of the top cells of mycelia were recorded. The ratio of clamp connection mycelium = the number of clamp connection/ mycelium; the surface area of the top cell of the mycelium $\left(\mu \mathrm{m}^{2}\right)=$ the length of the top cell of the mycelium $\times$ the diameter of the top cell of the mycelium; the branching frequency of the mycelium $\left(\mathrm{h}^{-1}\right)=$ the growth rate / the length of the top cell of the mycelium.

\section{Liquid culture of Auricularia cornea mycelia}

The Liquid medium contained potato $300 \mathrm{~g}$, glucose $20 \mathrm{~g}$, cornflour $10 \mathrm{~g}$, peptone $2 \mathrm{~g}$, potassium dihydrogen phosphate $2 \mathrm{~g}$, magnesium sulfate $1 \mathrm{~g}$, vitamin $\mathrm{B}_{1} 0.5 \mathrm{~g}$, 
water $1000 \mathrm{~mL}$. Different amounts of tannin mother liquor (sterilized, $5 \mathrm{~g} / \mathrm{L}$ ) were added to the sterilized liquid medium to make the different tannin concentrations in the liquid medium $0,0.05,0.1,0.15,0.2,0.25$ and $0.3 \mathrm{~g} / \mathrm{L}$, respectively. Each process was repeated three times. After cooling, six Auricularia cornea strains of the same age and size were added to each bottle. The cells were cultured and kept shaking in shaker for nine days at $150 \mathrm{rpm}$ and temperature was maintained at $24^{\circ} \mathrm{C}$.

Determination of dry weight of mycelium: The mycelium was baked at $60{ }^{\circ} \mathrm{C}$ to constant weight and weighed by electronic balance.

\section{Tannin stress on hypha culture of Auricularia cornea}

Liquid medium: The formula is mentioned in section Liquid Culture of Auricularia cornea Mycelia. When the liquid medium was cooled, the activated Auricularia cornea strain was inoculated and cultured in a shaker at $150 \mathrm{rpm}$ and $24{ }^{\circ} \mathrm{C}$ for 6 days. Different amounts of tannin mother liquor (sterilized, $5 \mathrm{~g} / \mathrm{L}$ ) were added to the liquid culture medium to make the tannin concentration in the medium 0 , $0.05,0.1,0.15,0.2,0.25$ and $0.3 \mathrm{~g} / \mathrm{L}$, respectively. Then they were cultured at $24{ }^{\circ} \mathrm{C}$ and sampled at $0,6,12,24,48$ and $72 \mathrm{~h}$, respectively.

\section{Preparation of crude enzyme solution}

The supernatant of $2 \mathrm{~mL}$ sample was centrifuged at $8000 \mathrm{rpm}$ and $4{ }^{\circ} \mathrm{C}$ for 10 min. It was a crude enzyme solution of Auricularia cornea. Stored in refrigerator at $-80{ }^{\circ} \mathrm{C}$.

\section{Determination of enzyme activity}

The enzyme activity was determined by kit method.

\section{Data analysis}

All test data were analyzed by Excel and IBM SPSS Statistics 24 software. 


\section{Results and analysis}

\section{Effect of tannin on mycelial growth of Auricularia cornea}

\section{Effect of tannin on plate culture mycelia}

Tannin concentration had a significant effect on the mycelial morphology of Auricularia cornea. The white and aerial hyphae grew vigorously in circular form on PDA medium having tannin conc. $0.05-0.1 \mathrm{~g} / \mathrm{L}$ (Fig q). The mycelium grew vigorously and formed the largest circle of mycelium on $0.1 \mathrm{~g} / \mathrm{L}$ tannin. When tannin concentration reached $0.2 \mathrm{~g} / \mathrm{L}$, the aerial hyphae was less developed, the hyphae was thinner and the hyphae circle was smaller in comparison to control $(0 \mathrm{~g} / \mathrm{L})$. With increased concentration of Tannin $0.3 \mathrm{~g} / \mathrm{L}$, the mycelium was weak, and the smallest mycelium circle was formed in the 7 th treatments.

The effect of tannin concentration on the mycelial growth rate of Auricularia cornea was significant. Mycelial growth rate was higher on tannin concentrations 0.05-0.10 g/L than control (0 g/L) (Fig 2). Higher mycelial growth was recorded on $0.1 \mathrm{~g} / \mathrm{L}$ concentration of tannin and increased $15.1 \%$ in comparison to control $(0 \mathrm{~g} / \mathrm{L})$. With the increased tannin concentration, mycelial growth was inhibited. When tannin concentration reached $0.3 \mathrm{~g} / \mathrm{L}$, the inhibition of mycelial growth was high and the mycelial growth rate was $63.0 \%$ lower than control $(0 \mathrm{~g} / \mathrm{L})$. We also had a look at the combined effect of tannins and iron $\left(\mathrm{Fe}^{3+}\right)$ on mycelial growth (Table 1).

\section{Effect of tannin on dry weight of hyphae in Liquid culture}

Tannin concentration had a significant effect on the dry weight of Auricularia cornea hyphae cultured in a liquid medium. The dry weight of mycelium decreased with increased tannin concentration (Fig 3). When tannin concentration was $0.05 \mathrm{~g} / \mathrm{L}$, the dry weight of mycelium decreased by $32.6 \%$ in comparison with control $(0 \mathrm{~g} / \mathrm{L})$. When tannin concentration increased from 0.25 to $0.3 \mathrm{~g} / \mathrm{L}$, the dry weight of mycelium significantly decreased from $71.8 \%$ to $77.7 \%$ respectively in comparison to control $(0 \mathrm{~g} / \mathrm{L})$. 


\section{Effect of tannins on the clamp connection ratio}

Tannin concentration can significantly affect the clamp connection ratio of mycelia under microscopy. As shown in Fig. 4, when the tannin concentration in the medium was $0.05-0.1 \mathrm{~g} / \mathrm{L}$, the clamp connection ratio showed an upward trend compared with the control $(0 \mathrm{~g} / \mathrm{L})$. When the tannin concentration was $0.1 \mathrm{~g} / \mathrm{L}$, the clamp connection ratio was the largest and increased by $13.2 \%$ compared with the control $(0 \mathrm{~g} / \mathrm{L})$. When tannin concentration increased to $0.15 \mathrm{~g} / \mathrm{L}$, the clamp connection ratio decreased gradually. When tannin concentration was $0.3 \mathrm{~g} / \mathrm{L}$, the clamp connection ratio under microscopy decreased by $51.5 \%$ compared with the control $(0$ $\mathrm{g} / \mathrm{L})$.

\section{Effect of tannin on mycelial microstructure}

The morphology of Auricularia cornea hyphae was different under different tannin concentrations, especially the frequency of mycelial branches directly related to the growth rate (Liu et al. 2017). The morphological responses of Auricularia cornea hyphae to tannin stress were different. During the period of mycelial growth under tannin stress, the mycelial branching rate of CK $(0 \mathrm{~g} / \mathrm{L})$ decreased with the increase of tannin concentration except that the mycelial branching rate of CK $(0.1$ $\mathrm{g} / \mathrm{L}$ ) was higher than CK (Table 1). The diameter of mycelial apical cells decreased by $7.7 \%, 18.0 \%, 25.6 \%$ and $33.3 \%$ at $0.15,0.2,0.25$ and $0.3 \mathrm{~g} / \mathrm{L}$, respectively; the length of mycelial apical cells did not change significantly; the surface area of mycelial apical cells decreased by $6.3 \%, 13.6 \%, 27.2 \%$ and $18.2 \%$ respectively. The mycelial branching rate decreased by $13.9 \%, 17.5 \%, 32.7 \%$ and $68.6 \%$ respectively; the above results showed that mycelial cells presented a comparative appearance with CK during high concentrations of tannin stress. Morphological changes gradually thinned. Among the four microscopic morphological indicators, the decrease in mycelial branching frequency was high among the four strains, which was an important indicator related to the growth rate of mycelium visible to the naked eye and the length of the top cell of the micro-mycelium. 


\section{Effects of tannin stress on antioxidant enzyme activity of mycelia}

\section{Effect of tannin stress on TBARS content}

The condensation reaction of MDA with thiobarbituric acid produces red products. TBARS content could be used as an index to measure lipid peroxidation and to assess the oxidative stress level of cell membrane (Kim et al. 2006). As shown in Fig.5, TBARS content in mycelia showed an overall upward trend with different tannin concentrations ( $24 \mathrm{~h}$ treatment). It indicated that tannin stress had a significant effect on TBARS content in mycelium. When tannin concentration reached $0.05 \mathrm{~g} / \mathrm{L}$, mycelial TBARS content increased by $26.5 \%$ compared with control $(0 \mathrm{~g} / \mathrm{L})$. When tannin concentration was $0.15 \mathrm{~g} / \mathrm{L}$, the TBARS content in mycelium reached the maximum level, and the TBARS content in mycelium increased by $45.6 \%$ in comparison with control $(0 \mathrm{~g} / \mathrm{L})$. When tannin concentration increased to $0.20 \mathrm{~g} / \mathrm{L}$, the TBARS content in mycelium tended to increase to a certain value.

As shown in Fig. 6, the TBARS content in mycelia showed an overall increasing trend with different tannin stress treatment time. At $48 \mathrm{~h}$, the TBARS content in mycelium reached the maximum level, which increased by $32.5 \%$ in comparison with control $(0 \mathrm{~h})$. TBARS content in mycelia decreased from $72 \mathrm{~h}$ to $24 \mathrm{~h}$ after treatment. This indicated that tannin stress had a time effect on TBARS content of Auricularia cornea hyphae.

\section{Effects of tannin stress on SOD activity}

Superoxide dismutase (SOD) is a class of enzymes containing metal coenzymes, which widely exists in organisms. Its function is to catalyze the disproportion reaction of superoxide anion radicals to produce hydrogen peroxide and oxygen, so as to achieve the purpose of scavenging superoxide anion radicals (Heath and Packer 1968). Fig. 7 showed that SOD activity of Auricularia cornea mycelia increased continuously within 72 hours of tannin stress treatment. At $72 \mathrm{~h}$, the SOD activity of mycelia was 6.1 times higher than that of control $(0 \mathrm{~h})$, indicating that tannin stress significantly 
increased the SOD activity of mycelia.

\section{Effect of tannin stress on POD activity}

Peroxidase (POD) widely exists in organisms. Its function is to catalyze the oxidation of various reductants involving hydrogen peroxide. The reaction formula is $\mathrm{RH}_{2}+\mathrm{H}_{2} \mathrm{O}_{2} \_2 \mathrm{H}_{2}+\mathrm{R}$ (Tian et al. 2001). Fig. 8 showed that POD activity of Auricularia cornea mycelia increased continuously within 72 hours of tannin stress treatment. POD activity of mycelium was 2.1 times higher than that of control $(0 \mathrm{~h})$ and reached its maximum level at $72 \mathrm{~h}$ after $6 \mathrm{~h}$ of tannin stress treatment. At this time, POD activity of mycelium was 4.8 times higher than that of control $(0 \mathrm{~h})$. The results showed that tannin stress could significantly increase POD activity.

\section{Effects of tannin stress on CAT activity}

Catalase (CAT) exists widely in animals and plants. In plants, CAT scavenges hydrogen peroxide produced during mitochondrial electron transfer, beta-fatty acid oxidation and photorespiration to prevent the damage of reactive oxygen species to plants (Yang and Poovaiah 2002). Figure 9 showed that within 72 hours of tannin stress treatment the C AT activity of Auricularia cornea decreased.

The CAT activity of hyphae showed a trend of decreasing first, then rising to the highest and then declining. Within $0-12 \mathrm{~h}$ of tannin stress treatment, the CAT activity of mycelium decreased gradually. At $12 \mathrm{~h}$ of treatment, the C AT activity of mycelium decreased by $44.9 \%$ compared with that of control $(0 \mathrm{~h})$. The CAT activity of mycelia reached the maximum at $24 \mathrm{~h}$, and increased by $90.1 \%$ as compared with the control $(0$ h). After 48 hours, the CAT activity of mycelium decreased again, and at 72 hours, the C AT activity of mycelium decreased by $19.6 \%$ compared with that of control ( 0 hours).

\section{Discussion}

The Auricularia cornea mycelial growth rate on PDA medium with different 
concentrations of tannin was the following: lower concentration promoted mycelial growth while higher concentration inhibited mycelial growth. When the tannin concentration in PDA medium exceeded $0.1 \mathrm{~g} / \mathrm{L}$, high concentration of tannin could inhibit mycelial growth. In the experiment of determining dry weight of Auricularia cornea mycelium in liquid tannin culture, the dry weight of Auricularia cornea mycelium decreased with increased tannin concentration, showed a significant negative correlation. The effect of tannin on Auricularia cornea mycelium was different in plate and liquid shaking flask. It may be that the area of contact medium containing tannin was different, and the mycelium could only grow on the surface of PDA medium containing tannin. The mycelium grew in liquid medium containing tannin, and the contact surface area was larger than that in plate culture. Experiments showed that the higher the tannin concentration, the longer the tannin treatment time, the stronger the inhibition of Auricularia cornea mycelial cells. Our results of present study are supported with the findings of Fan et al., (2006) who found that lower concentration of Tannin under $100 \mathrm{mg} / \mathrm{L}$ in the medium stimulated the growth of mycelia Pleurotus sp. but had negative effect above 500 and $1000 \mathrm{mg} / \mathrm{L}$. Similarly Cheo (1982) reported that mycelial growth of A. mellea was high on low tannin concentration $(0.001 \% \mathrm{w} / \mathrm{v})$ and low growth was recorded on high $(0.05 \% \mathrm{w} / \mathrm{v})$ concentration of tannin Catalase (CAT) and superoxide dismutase (SOD), peroxidase (POD) together constitute an antioxidant enzyme protection system (ZHANG Kun-sheng and TIAN Hui-lin 2007). These enzymes could scavenge free radicals and peroxides in cells. Thus, the accumulation of free radicals and superoxide can be reduced, and the damage to organisms could be reduced (Tian et al. 2001, ZHANG Kun-sheng and TIAN Hui-lin 2007). The results showed that the induction of tannin to antioxidant enzymes was not only related to the concentration but also closely related to the stress time. It was generally believed that the higher the tannin concentration, the longer the stress time, the greater the damage to biological cells.

This study found that tannin stress-induced the increase of lipid peroxidation in mycelial cells, with significant concentration and time effects ( $\mathrm{Lu} 2015)$. This was 
similar to the response of Pleurotus ostreatus cells to heavy copper ( $\mathrm{Fu}$ et al. 2010). The phenolic hydroxyl groups of tannins were easily oxidized by oxygen in the air, and the oxidation speed was accelerated in the aqueous state, resulting in discoloration. Phenolic hydroxyl groups could also react with free radicals to form polyphenol free radicals. Tannin also reduced oxygen content in the environment through reduction. According to the experimental data analysis, TBARS in mycelia increased under tannin stress, indicating that the oxidative stress level of cell membrane was high; SOD and POD activities were rising all the time, indicating that a large number of superoxide anion free radicals were produced and the oxidative reaction involving hydrogen peroxide was increased, and the mycelium needed to enhance SOD and POD activities in order to adapt to adverse environmental conditions. According to the experiment of heavy copper stress on Pleurotus ostreatus, tannin stress leads to hydrogen peroxide accumulation at the beginning and inhibits CAT activity (Lu 2015). After a period of adaptation, CAT responds to tannin stress and enzymes activity increases. However, its self-regulation ability was poor, and then its activity decreased again. The response of different antioxidant enzymes to tannin stress was different from that of the whole antioxidant enzymes system.

\section{Abbreviations}

CK, control; SOD, Superoxide dismutase; DAPI, 4',6-diamidino-2-phenylindole; PBS; PDA, potato dextrose agar; TBARS MDA malonaldehyde; POD, Peroxidase; CAT Catalase;

\section{Authors' contributions}

ZZ and DYF conceived and designed the experiments, performed the experiments, analyzed the data, wrote the paper, prepared figures and tables; BS and FHT conceived and designed the experiments; $\mathrm{CZ}$ and $\mathrm{CWJ}$ performed the experiments; CTL and YL conceived and designed the experiments, analyzed the data, contributed reagents/materials/analysis tools, reviewed drafts of the paper. These authors contributed equally to this work, All authors read and approved the final manuscript. 


\section{Author details}

${ }^{1}$ Engineering Research Center of Edible and Medicinal Fungi, Ministry of Education, Jilin Agricultural University, Changchun 130118,People's Republic of China. ${ }^{2}$ International Cooperative Research Center of China for New Germplasm Breeding of Edible Mushroom, Ministry of Science and Technology.

\section{Acknowledgments}

We thank Huang Jing for her help with the experimental techniques.

\section{Competing interests}

The authors declare that they have no competing interests.

\section{Availability of data and materials}

The datasets supporting the conclusions of this article are included in the article.

\section{Ethics approval and consent to participate}

This article does not contain studies with human participants or animals performed by any of the authors.

\section{Funding}

This work was supported by National Key Research and Development Program of China (2017YFD0601002), University S \& T Innovation Platform of Jilin Province for Economic Fungi (\#2014B-1) and, Special Fund for Agro-scientific Research in the Public Interest (201503137).

\section{References}

Cheo PC. (1982) Effects of Tannic Acid on Rhizomorph Production by Armillaria mellea[J].. Phytopathology. 72: 676-679, doi: 10.1094/phyto-77-676.

Fu CM, Huang KL, Wang Z. (2010) The Properties and Applications of Vegetable Tannin[J]. Enterp Sci Technol Dev. 22: 57-60

He X., Wang G. (1996) The Commercial Cultivation of Auricularia cornea by Using Sawdust of Pine and Chinese Fir Tree[J]. Edible Fungi. 6: 21-22.

Heath RL, Packer L. (1968) Photoperoxidation in isolated chloroplasts. I. Kinetics and stoichio metry of fatty acid peroxidation[J]. Arch Biochem Biophys. 125: 189-198, doi: 10.1016/00 03-9861(68)90654-1.

Kim IS, Moon HY, Yun HS, Jin I. (2006) Heat shock causes oxidative stress and induces a variety of 
cell rescue proteins in Saccharomyces cerevisiae KNU5377[J]. J Microbiol. 44: 492-501.

Liu XM, Wu XL, Chen Q, Chou ZH, Zhang J., C.Y. H. (2017) Effects of heat stress on Pleurotus eryngii mycelial growth and its resistance to Trichoderma asperellum[J]. Junwu Xuebao. 36: $1566-1574$.

Lu J. (2015) Effects of copper on growth and development of Pleurotus ostreatus[D]. Henan Agric Univ.

Ren X. (1980) Dendrology [M]. China For Publ House, Beijing, China. 6-75.

Tian G, Li H, Qiu W. (2001) Advances on research of plant peroxidases[J]. 19: 332-344.

Wang Y, Wang C, Guo M. (2019) Effects of ultrasound treatment on extraction and rheological properties of polysaccharides from Auricularia cornea var. Li. Molecules. Doi: 10.3390/m olecules24050939.

Zhang Kun-sheng, Tian Hui-lin. (2007) Research and function of catalase in organism[J]. Food Sci Technol. 1:8-11.

\section{Legends}

\section{Table legends}

Table 1. Tannin and Fe3+ effect on mycelium

Table 2: Influences of tannins stress on the hyphal morphology for $A$. cornea

\section{Figure legends}

Fig. 1 Photos of A. cornea on different concentrations of tannins PDA medium

Fig. 2 Effect of different concentrations of tannins on mycelial growth rate of $A$. cornea in PDA medium

Fig. 3 Effect of different concentrations of tannins on mycelial dry-weighted of $A$. cornea

Fig. 4 Effects of concentrations of tannins on the clamp-connection hyphae ration of $A$. cornea

Fig. 5 Effect of different concentrations of tannins on TBARS content of mycelial of $A$. cornea

Fig. 6 Effect of tannins on TBARS content of mycelial of $A$. cornea

Fig. 7 Effect of tannins on SOD activity of mycelial of $A$. cornea

Fig. 8 Effect of tannins on POD activity of mycelial of $A$. cornea

Fig. 9 Effect of tannins on CAT activity of mycelial of $A$. cornea 
Figures
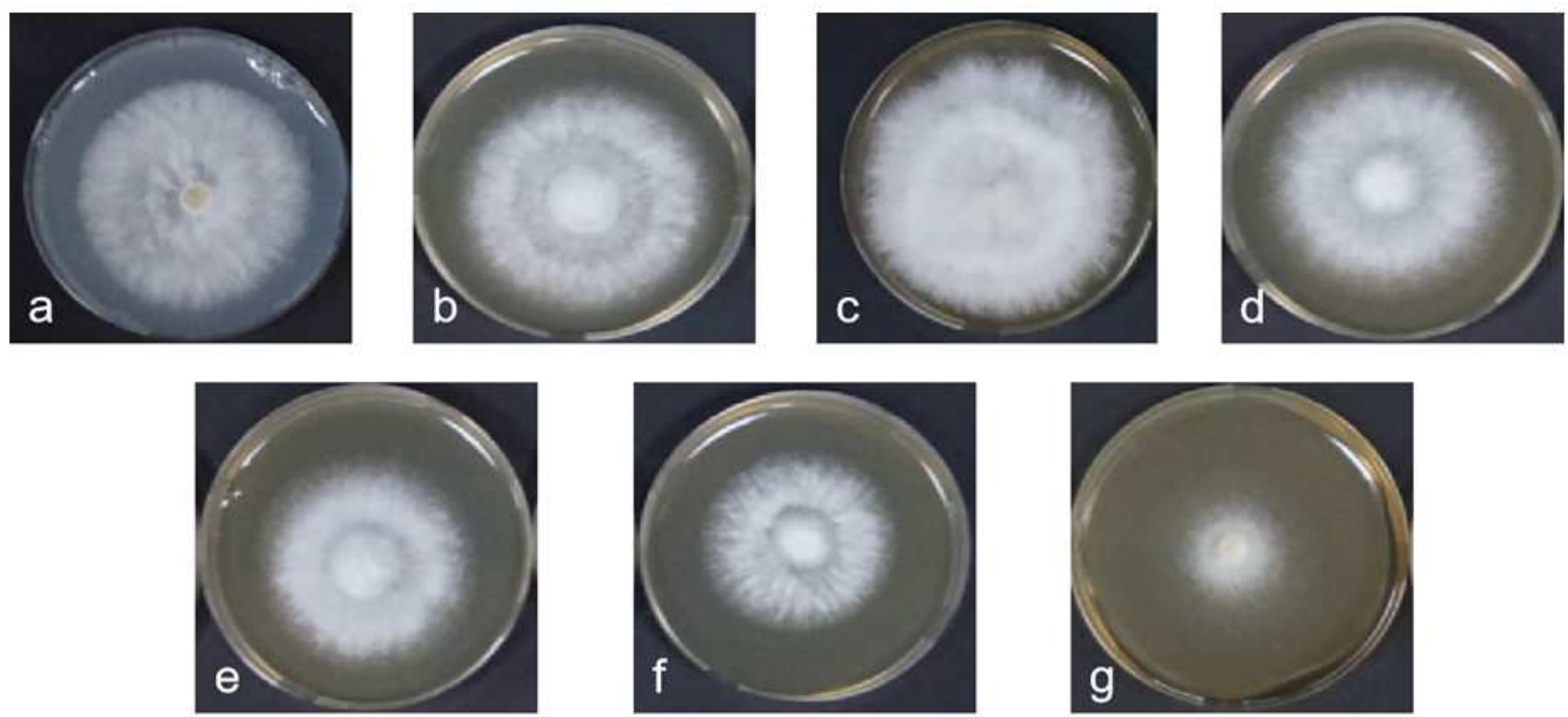

Figure 1

Photos of A. cornea on different concentrations of tannins PDA medium Note: Photos a, b, c, d, e, f, g tannin concentrations were $0,0.05,0.1,0.15,0.2,0.25$ and $0.3 \mathrm{~g} / \mathrm{L}$, respectively.

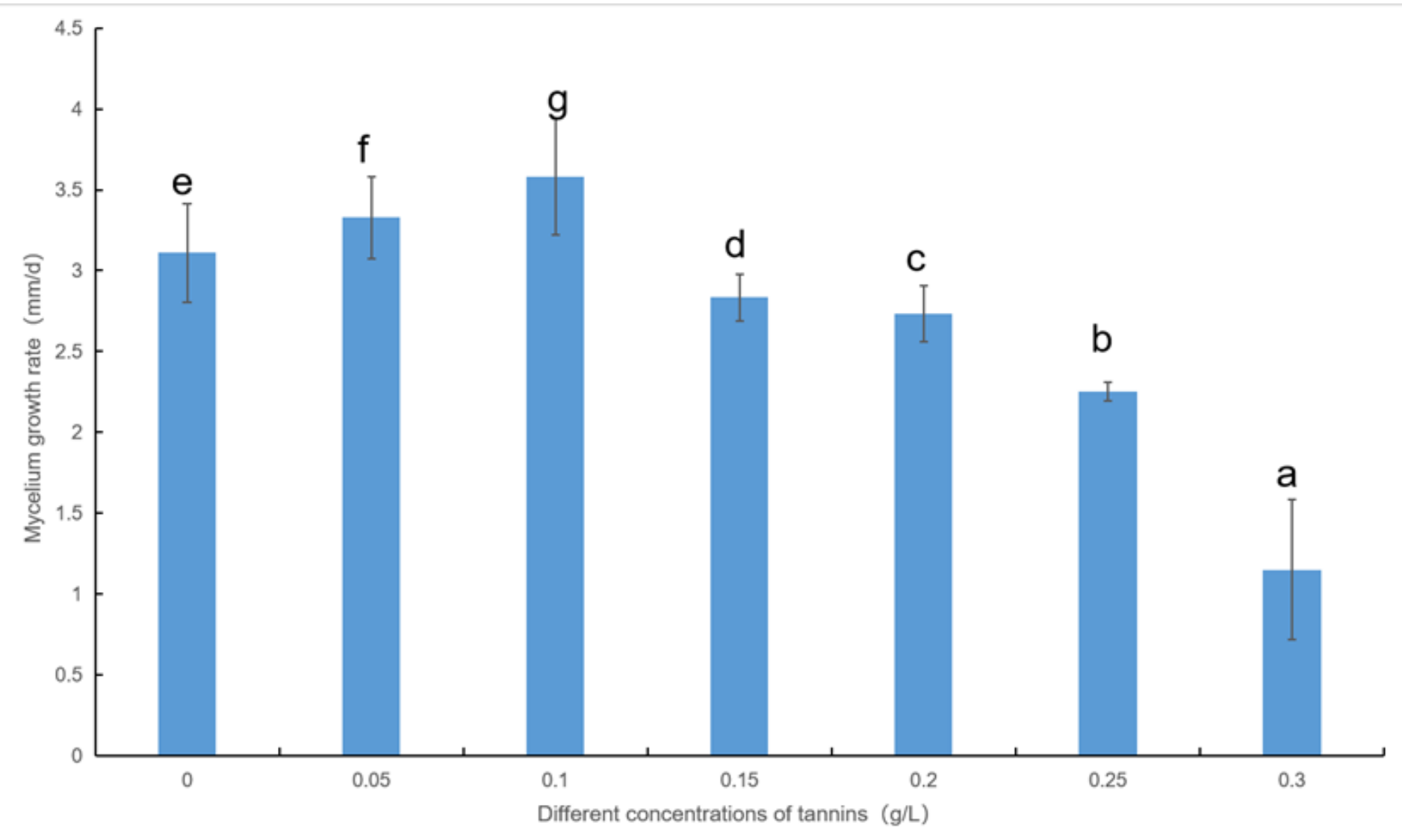


Figure 2

Effect of different concentrations of tannins on mycelial growth rate of A. cornea in PDA medium

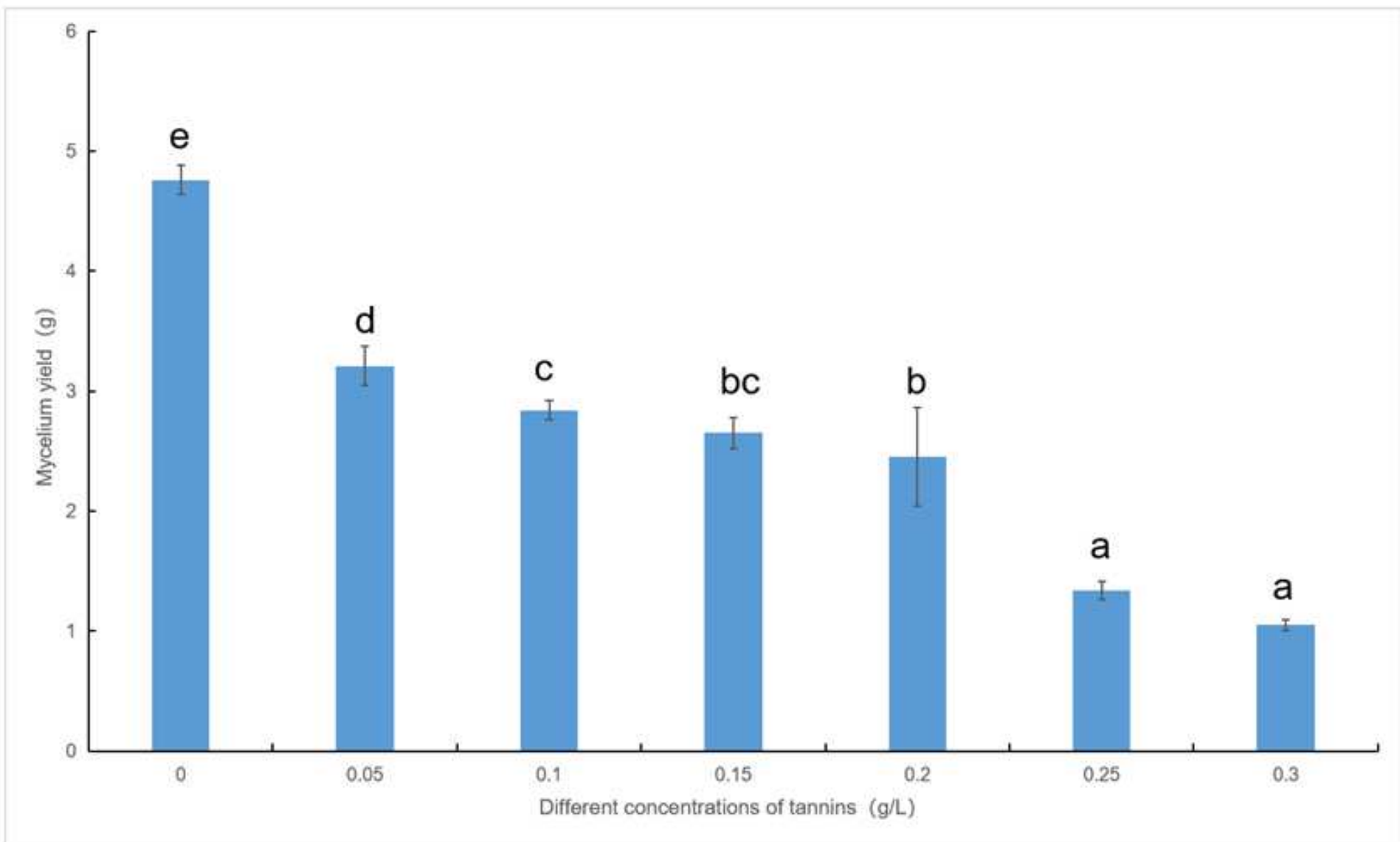

Figure 3

Effect of different concentrations of tannins on mycelial dry-weighted of A. cornea 


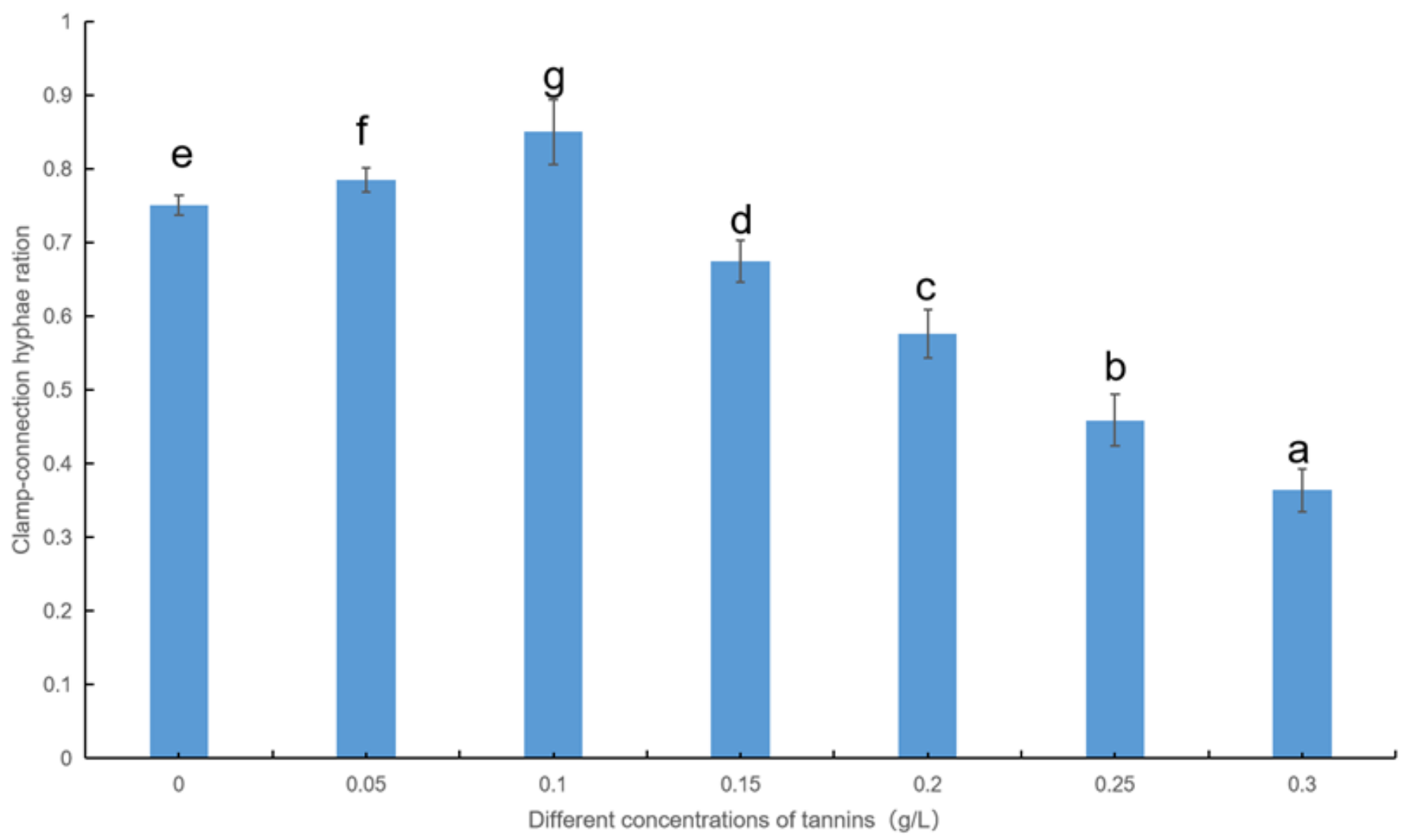

Figure 4

Effects of concentrations of tannins on the clamp-connection hyphae ration of $A$. cornea 


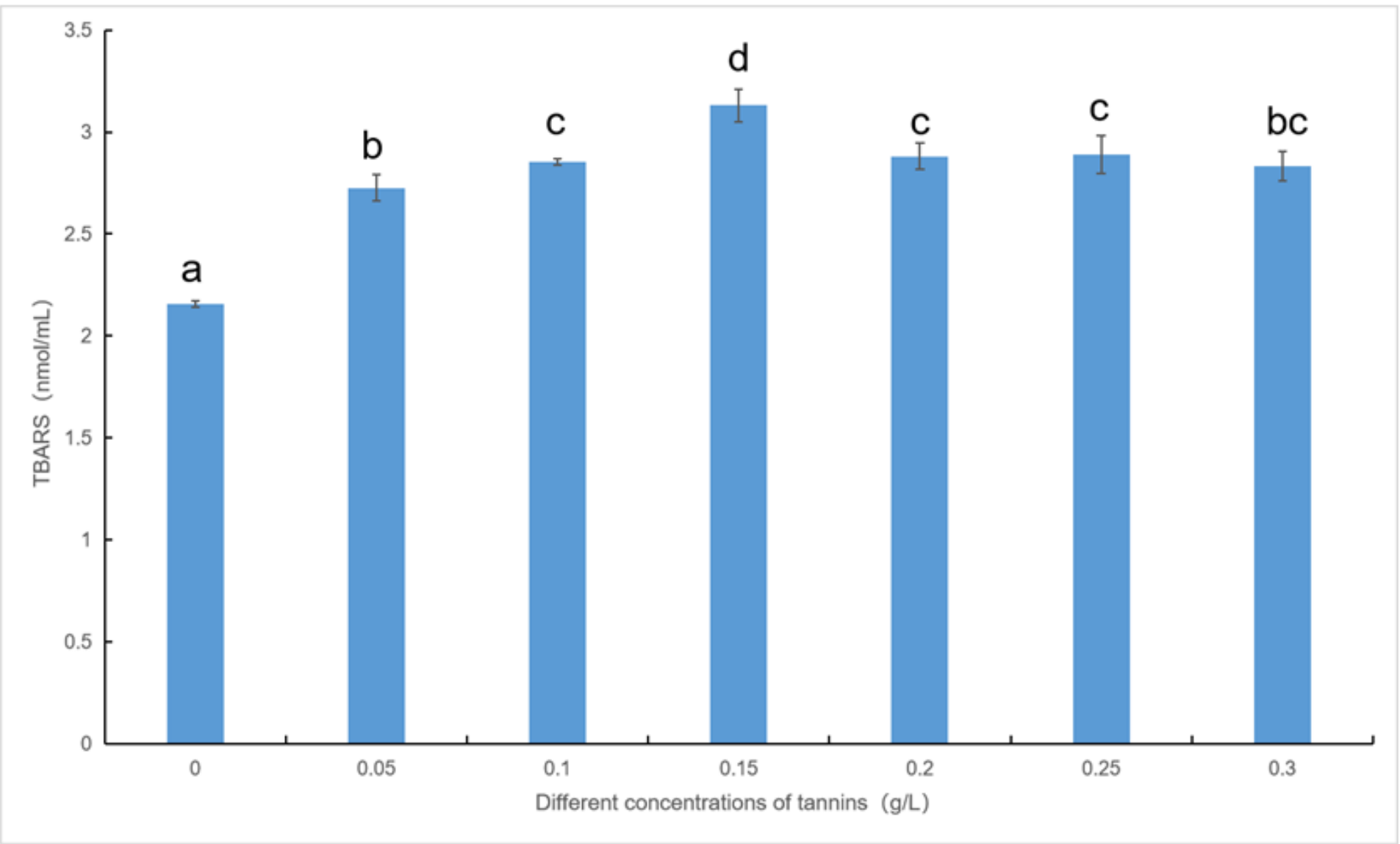

Figure 5

Effect of different concentrations of tannins on TBARS content of mycelial of A. cornea 


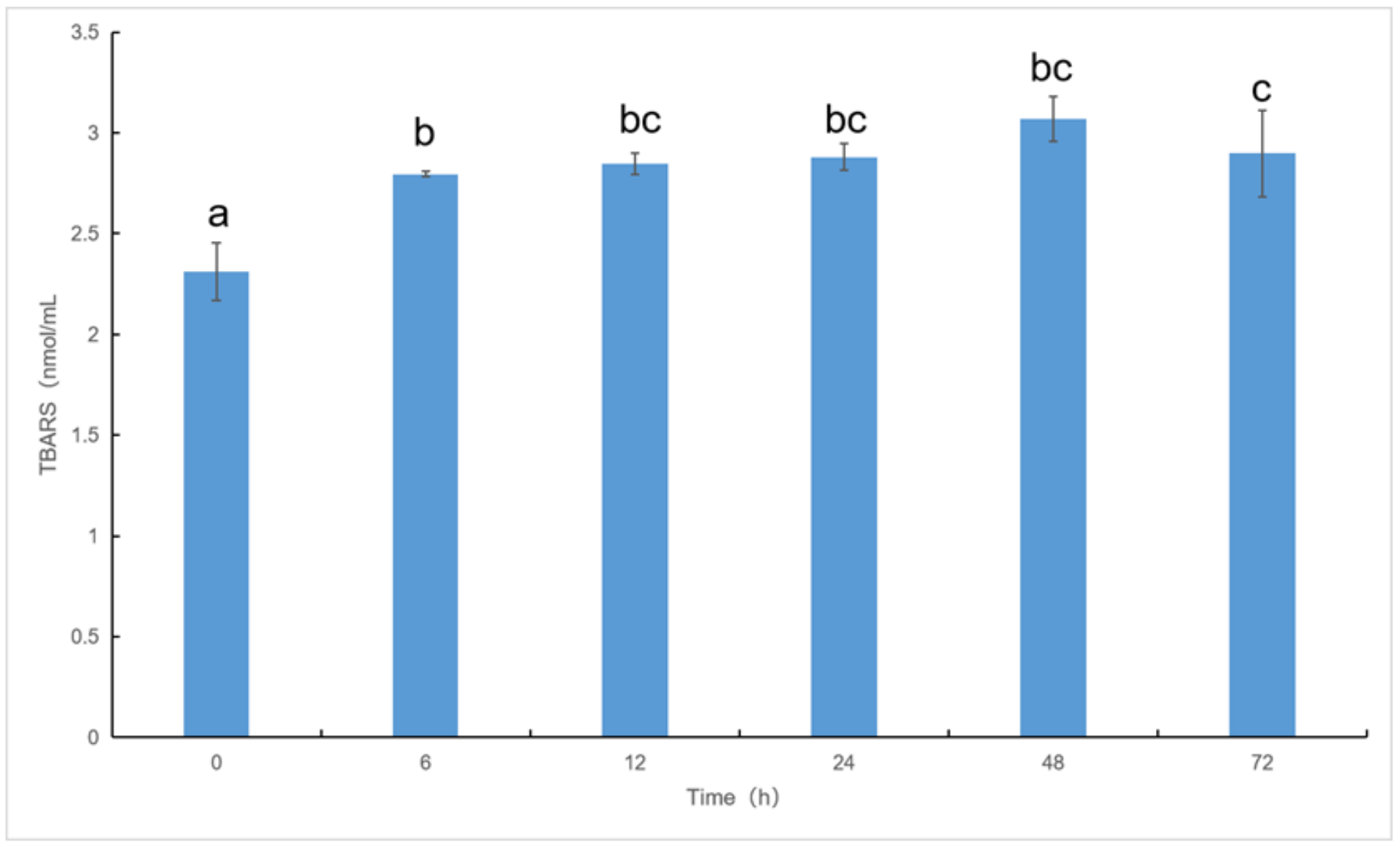

Figure 6

Effect of tannins on TBARS content of mycelial of A. cornea 


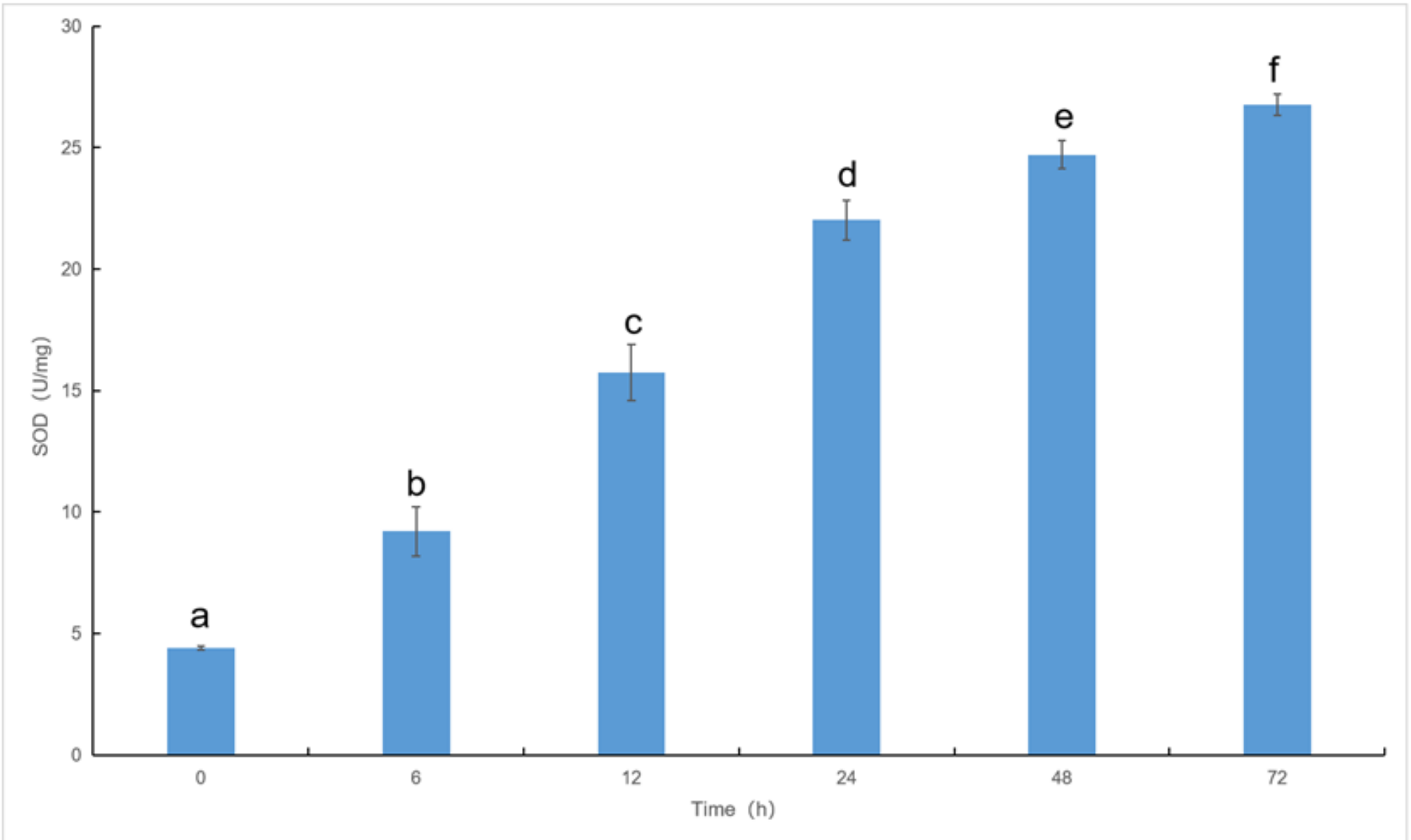

Figure 7

Effect of tannins on SOD activity of mycelial of A. cornea 


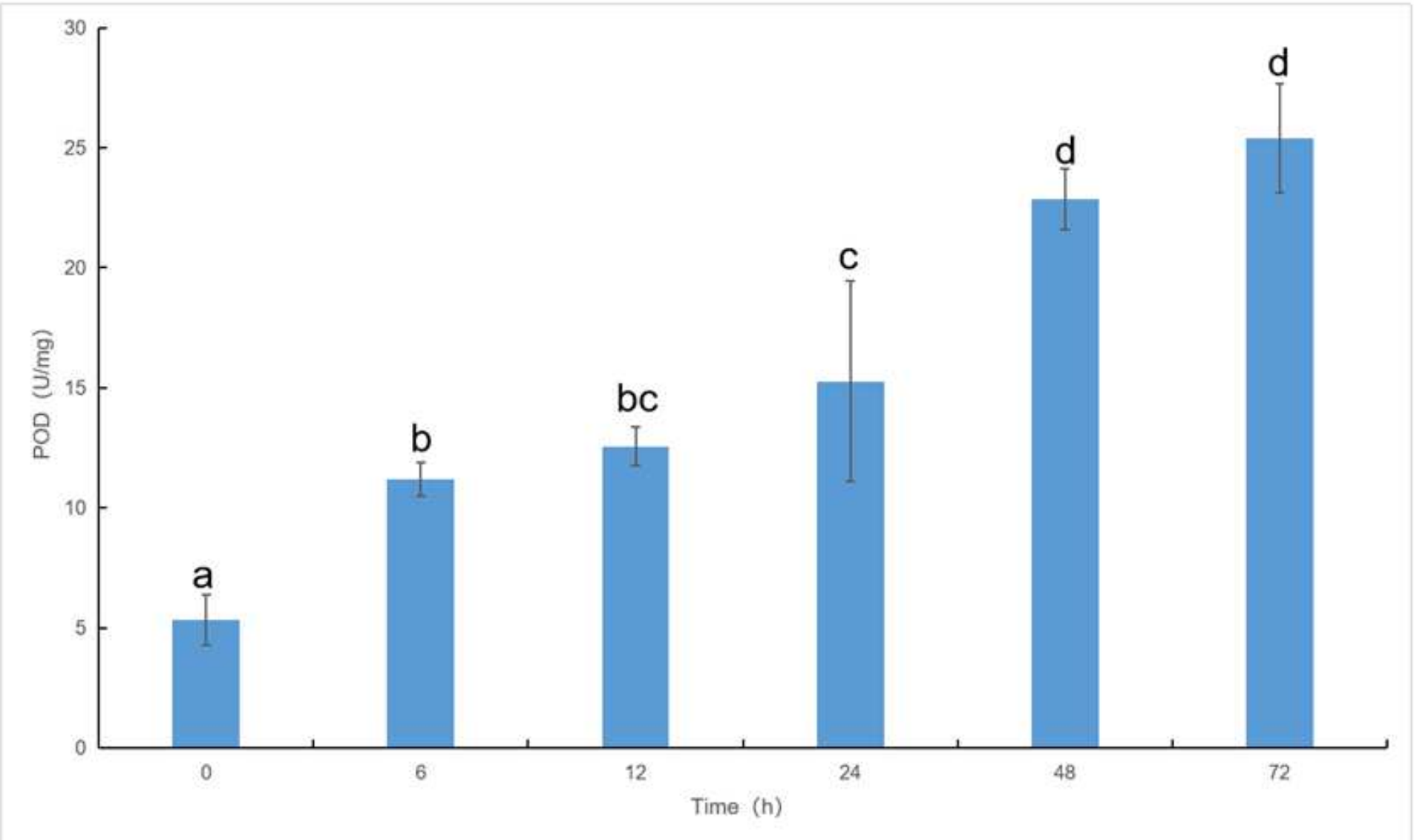

Figure 8

Effect of tannins on POD activity of mycelial of A. cornea 


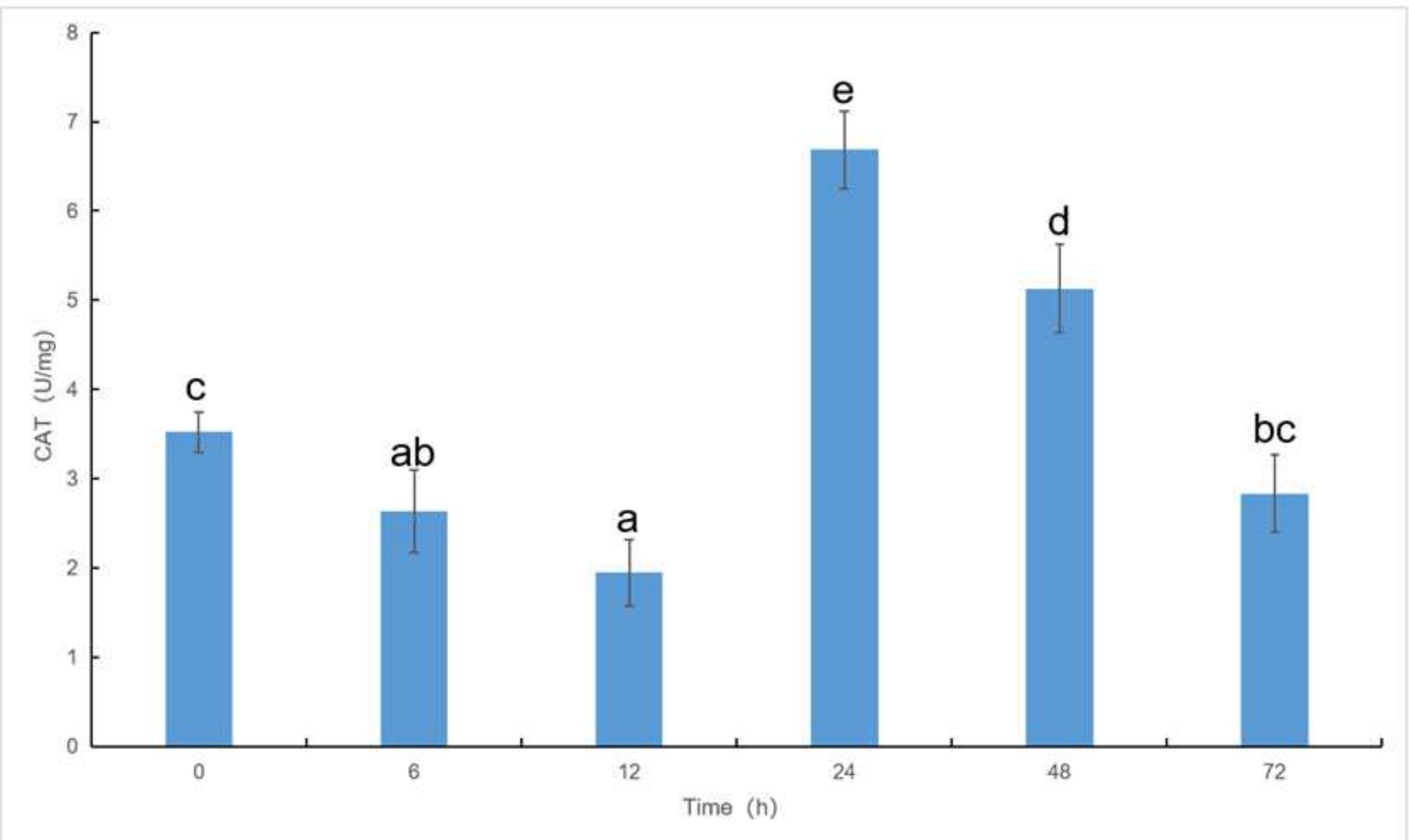

Figure 9

Effect of tannins on CAT activity of mycelial of A. cornea

\section{Supplementary Files}

This is a list of supplementary files associated with this preprint. Click to download.

- Table.pptx

- Table.pptx 